\title{
Article \\ Affine Differential Geometric Control Tools for Statistical Manifolds
}

\author{
Iulia-Elena Hirica $^{1, *,+(\mathbb{D}}$, Cristina-Liliana Pripoae ${ }^{2,+} \mathbb{D}$, Gabriel-Teodor Pripoae ${ }^{1,+} \mathbb{D}$ and Vasile Preda ab $^{1,+}$ \\ 1 Faculty of Mathematics and Computer Science, University of Bucharest, Academiei 14, \\ RO-010014 Bucharest, Romania; gpripoae@fmi.unibuc.ro (G.-T.P.); preda@fmi.unibuc.ro (V.P.) \\ 2 Department of Applied Mathematics, The Bucharest University of Economic Studies, Piata Romana 6, \\ RO-010374 Bucharest, Romania; cristinapripoae@csie.ase.ro \\ * Correspondence: ihirica@fmi.unibuc.ro \\ + These authors contributed equally to this work.
}

Citation: Hirica I.-E.; Pripoae C.-L.; Pripoae, G.-T.; Preda, V. Affine Differential Geometric Control Tools for Statistical Manifolds. Mathematics 2021, 9, 1654. https://doi.org/ $10.3390 /$ math 9141654

Academic Editor: Vlad Stefan Barbu

Received: 27 June 2021

Accepted: 12 July 2021

Published: 14 July 2021

Publisher's Note: MDPI stays neutral with regard to jurisdictional claims in published maps and institutional affiliations.

Copyright: () 2021 by the authors. Licensee MDPI, Basel, Switzerland. This article is an open access article distributed under the terms and conditions of the Creative Commons Attribution (CC BY) license (https:// creativecommons.org/licenses/by/ $4.0 /)$.
Abstract: The paper generalizes and extends the notions of dual connections and of statistical manifold, with and without torsion. Links with the deformation algebras and with the Riemannian Rinehart algebras are established. The semi-Riemannian manifolds admitting flat dual connections with torsion are characterized, thus solving a problem suggested in 2000 by S. Amari and H. Nagaoka. New examples of statistical manifolds are constructed, within and beyond the classical setting. The invariant statistical structures on Lie groups are characterized and the dimension of their set is determined. Examples for the new defined geometrical objects are found in the theory of Information Geometry.

Keywords: statistical manifold; dual connections; Fisher metric; Gibbs entropy; invariant connections on Lie groups; Information Geometry; dually flat connections; deformation algebras; Riemannian Rinehart algebras; bi-algebras; control tools

\section{Introduction}

A triple $(M, g, \nabla)$ is called statistical manifold if $(M, g)$ is a semi-Riemannian manifold and $\nabla$ is a torsion-free affine connection on $M$ such that

$$
\left(\nabla_{Z} g\right)(X, Y)=\left(\nabla_{Y} g\right)(X, Z)
$$

This notion was defined by S. Amari in [1], as a geometrical model for some facts in Statistics: $M$ is a parameters space of distributions of probability, $g$ is the Rao-Fisher metric deduced from the Bolzmann-Gibbs-Shannon entropy function, and $\nabla$ is a tool for asymptotic estimations.

The geometrization based on both a semi-Riemannian metric and an affine connection was already used (until now, without great succes) in different attempts to unify relativistic gravity models with electromagnetism ones (Weyl, Eddington, Einstein, Kaluza, etc., in the first half of the 20th century; see in [2] for a recent review). Instead, in Statistics, the model was considered important and fruitful. Today it constitutes a modern and promising area of active research (see, for example, in [3-6]).

Two connections $\nabla^{1}$ and $\nabla^{2}$ are dual in $(M, g)$ if

$$
X g(Y, Z)-g\left(\nabla_{X}^{1} Y, Z\right)-g\left(Y, \nabla_{X}^{2} Z\right)=0 .
$$

From a differential geometric point of view, the dualistic structure first generalizes somehow the invariance of the inner product under parallel translation through metric connections. Moreover, the existence of a dually flat structure on a manifold points out some topological and geometrical properties of the manifold. The notion traces back to Norden and was adapted on statistical manifolds, where remarkable families of dual 
connections contain information about the dualistic properties of exponential families of probability distributions [7].

This initial (and already classical) setting was generalized in several ways. We point out here only a direction opened by Kurose and Matsuzoe, who considered statistical manifolds with non-symmetric connections $\nabla$ [8-10], intended for quantum field theories. As statistical manifolds have close relations to the geometry of affine immersions, statistical manifolds admitting torsion have relations to the geometry of affine distributions.

In this paper, we review in a creative manner the fundamentals of dual connections and of statistical manifolds and we give new examples (Sections 2 and 4). The families of these new examples depend on many "parameters" and thus are susceptible to fit in various applications. We establish "controls" over the parameters manifold $M$ and its various affine modules of connections. These "controls" are provided by deformation algebras defined by the difference of two connections, or by some Riemannian Rinehart bi-algebras associated to the Riemannian metric and to the canonical Lie-Rinehart algebra of the manifold. The deformation algebras were intensively studied during 1970-1990, as a natural translation between algebraic and differential geometric properties of differentiable manifolds. The Riemannian Rinehart structures are more recent and constitute a promising area of research. In Section 2, we give some hints for the respective literature related to both these algebraic objects. We show that arbitrary pairs of dual connections are determined by pairs of a metric connection and an arbitrary connection, or by triples formed by a metric connection, an arbitrary connection and a function (thus generalizing the pairs of the so called $\alpha$-connections).

In Section 3, we prove formulas for the main invariants associated to the dual connections. We determine links between their Bianchi identities and express the Jacobi equation for geodesics in terms of dual connections.

In Section 4, some very general families of statistical manifolds are defined, depending on many parameters. Here, we characterize the semi-Riemannian manifolds admitting flat dual connections with torsion, thus solving a problem suggested in [7].

In Section 5, we define nine new families of statistical manifolds, denoted $\mathrm{SMAT}_{1}, \ldots$, $\mathrm{SMAT}_{9}$, which generalize the known ones, by using some special hypothesis on the curvature and on the tensor vector fields.

In Section 6, we determine how many independent bi-invariant statistical structures may exist on a compact Lie group and how many independent left invariant statistical structures may exist on an arbitrary Lie group.

Section 7 is devoted to some examples of statistical manifolds, in particular frameworks from Information Geometry.

\section{Dual Connections and Controls over Some Affine Modules of Connections}

Let $(M, g)$ be a semi-Riemannian manifold with the Levi-Civita connection $\nabla^{0}$. We denote by $\mathcal{C}(M)$ and $\mathcal{C}_{s}(M)$ the sets of affine connections and of symmetric (i.e., torsionfree) connections on $M$, respectively, endowed with the canonical structure of affine $\mathcal{F}(M)$ module. We define

$$
\begin{gathered}
\mathcal{C}_{m}(M, g):=\left\{\nabla \in \mathcal{C}(M) \mid\left(\nabla_{Z} g\right)(X, Y)=0 \text { for all } X, Y, Z \in \mathcal{X}(M)\right\}, \\
\mathcal{C}_{c}(M, g):=\left\{\nabla \in \mathcal{C}(M) \mid\left(\nabla_{Z} g\right)(X, Y)=\left(\nabla_{Y} g\right)(X, Z) \text { for all } X, Y, Z \in \mathcal{X}(M)\right\}
\end{gathered}
$$

and $\mathcal{C}_{s c}(M, g):=\mathcal{C}_{s}(M) \cap \mathcal{C}_{c}(M, g)$. As $\left\{\nabla^{0}\right\}=\mathcal{C}_{s}(M) \cap \mathcal{C}_{m}(M, g)$, we have the following inclusions of (non-void) affine submodules

$$
\mathcal{C}_{s c}(M, g) \subset \mathcal{C}_{s}(M) \subset \mathcal{C}(M), \mathcal{C}_{s c}(M, g) \subset \mathcal{C}_{c}(M, g) \subset \mathcal{C}(M), \mathcal{C}_{m}(M, g) \subset \mathcal{C}_{c}(M, g) .
$$

Remark 1. (i) Each connection $\nabla \in \mathcal{C}(M)$ may be uniquely written as $\nabla=\nabla^{0}+A$, where $A \in \mathcal{T}_{2}^{1}(M)$ relates to the torsion tensor field $T^{\nabla}$ by the relation

$$
T^{\nabla}(X, Y)=A(X, Y)-A(Y, X) .
$$


We have the complete determination of the connection $\nabla$ by the (1,2)-tensor field $A$, and, mutatis mutandis, the determination of the affine module $\mathcal{C}(M)$ by its direction, the real vector space $\mathcal{T}_{2}^{1}(M)$. We may interpret the semi-Riemannian geometry of $\left(M, g, \nabla^{0}\right)$ as a reference point and "vary" it by affine geometries $(M, \nabla)$, acting through the "control" $A$. Moreover, once $A$ is fixed, $\mathcal{X}(M)$ gets a structure of $\mathcal{F}(M)$-algebra, called the deformation algebra of the pair $\left(\nabla, \nabla^{0}\right)$, by the multiplication $X \cdot Y:=A(X, Y)$. Translations between the algebraic properties of these deformation algebras and the geometric properties of the ambient manifold were extensively studied (see, for example, in [11-13] and the references therein).

We must point out here alternative invariants, also studied in the literature: the "cubic forms" $C_{1}(X, Z, Y):=\left(\nabla_{X} g\right)(Y, Z)$ and $C_{2}(X, Z, Y):=g(X, A(Y, Z))$; we shall not use them in our paper.

(ii) We have the following characterizations:

$$
\begin{gathered}
\mathcal{C}_{m}(M, g)=\left\{\nabla^{0}+A \mid g(A(X, Y), Z)+g(A(X, Z), Y)=0\right\}, \\
\mathcal{C}_{c}(M, g)=\left\{\nabla^{0}+A \mid g(A(X, Y), Z)+g(A(X, Z), Y)=\right. \\
=g(A(Z, Y), X)+g(A(Z, X), Y)\}, \\
\mathcal{C}_{s c}(M, g)=\left\{\nabla^{0}+A \mid g(A(X, Z), Y)=g(A(Y, Z), X), A(X, Y)=A(Y, X)\right\} .
\end{gathered}
$$

(iii) A Riemannian Rinehart space is a Lie-Rinehart algebra endowed with a "Riemannian metric", i.e., a musical (generalized) scalar product (see in [14] for details). This construction establishes a purely algebraic framework for many properties which are commonly studied in Riemannian geometry, by analytic and geometric tools.

In particular, on the Lie-Rinehart algebra $(\mathcal{F}(M), \mathcal{X}(M))$ [14], we get a canonical structure of Riemannian Rinehart space, induced by the (generalized) scalar product $<,>$, canonically associated to the Riemannian metric $g$.

Fix a connection $\nabla=\nabla^{0}+A \in \mathcal{C}(M)$, with the (fixed) control $A$. Define, as above, the multiplication $X \cdot Y:=A(X, Y)$. It follows that on $(\mathcal{F}(M), \mathcal{X}(M),<,>)$ we get an additional algebra structure, which combines the properties of the deformation algebra $(\mathcal{X}(M), \cdot)$ with those from the Lie-Rinehart algebra $(\mathcal{F}(M), \mathcal{X}(M))$. We believe that these bi-algebras $(\mathcal{F}(M), \mathcal{X}(M),<,>, \cdot)$ deserve a closer attention of their own.

In what we are concerned here, we restrain to the following.

Theorem 1. Let $(M, g)$ be a Riemannian manifold and $\nabla=\nabla^{0}+A$ a connection on $M$. Then, $\nabla \in \mathcal{C}_{c}(M, g)$ if and only if the Riemannian Rinehart bi-algebra $(\mathcal{F}(M), \mathcal{X}(M),<,>, \cdot)$ satisfies the compatibility condition

$$
<X \cdot Y, Z>-<Z \cdot Y, X>=<Z \cdot X-X \cdot Z, Y>
$$

We read this formula as a mutual determinancy, which express the obstruction to "selfadjointness", in the left side, through the obstruction to commutativity, in the right side.

Remark 2. (i) Consider the affine transformation $\Phi: \mathcal{C}(M) \rightarrow \mathcal{C}(M)$, which associates to each $\nabla \in \mathcal{C}(M)$ the affine connection $\nabla^{*}:=\Phi(\nabla)$, given by

$$
g\left(X, \nabla_{Z}^{*} Y\right)=Z g(X, Y)-g\left(\nabla_{Z} X, Y\right)
$$

The connection $\nabla^{*}$ is called the dual of $\nabla[1,7]$; the relation of duality is an equivalence one, as $\Phi$ is an involution. Obviously, $\nabla^{0}$ is (the only) self-dual connection, as the unique fixed point of $\Phi$. The transformation $\Phi$ depends on the Riemannian metric only.

(ii) Suppose $\nabla=\nabla^{0}+A$ and denote $A^{\prime}, ' A \in \mathcal{T}_{2}^{1}(M)$ the adjoint operators (at the right and at the left, respectively) given by

$$
g\left(X, A^{\prime}(Z, Y)\right)=g(Y, A(Z, X)), g\left(X,{ }^{\prime} A(Y, Z)\right)=g(Y, A(X, Z)) .
$$


We have also a direct relation between $A^{\prime}$ and 'A, which allows us to study only one of these operators, namely,

$$
g\left(X, A^{\prime}(Z, Y)\right)=g(Z, A(Y, X))
$$

Obviously, $A(X, Y)=A^{\prime}(Y, X)$ if and only if $A$ is commutative.

We have $\nabla^{*}:=\nabla^{0}+A^{*}$, where $A^{*}:=-A^{\prime}$. The deformation algebras $(\mathcal{X}(M), A)$ and $\left(\mathcal{X}(M), A^{*}\right)$ are equivalent, i.e., contain the same (algebraic and) geometric information. Obviously, $A^{\prime}=A$ if and only if $\nabla^{0}=\frac{1}{2}\left(\nabla+\nabla^{*}\right)$.

(iii) Another interesting deformation algebra is $\left(\mathcal{X}(M), A+A^{\prime}\right)$, which measures another obstruction for $\nabla$ to coincide with $\nabla^{*}$ (i.e., $\nabla=\nabla^{*}$ if and only if $A+A^{\prime}=0$ if and only if $\left.\nabla_{X} g=0\right)$.

(iv) The tensor fields $A^{*}, A^{\prime}$, and 'A (and their associated deformation algebras, or their associated Riemannian Rinehart bi-algebras) act as controls over the affine modules of special connections previously studied. The information they carry with is, of course, redundant and may be translated and simplified, following the context.

(v) Let $\left(\nabla, \nabla^{*}\right)$ be dual connections and $\tilde{\nabla}:=\frac{1}{2} \nabla+\frac{1}{2} \nabla^{*}$ their mean connection. It is well known $[7,10]$ that $\tilde{\nabla} \in \mathcal{C}_{m}(M)$. We remark also that $\tilde{T}=\frac{1}{2} T-\frac{1}{2} T^{\prime}$.

It is interesting that we have also a strong converse statement (inspired by a suggestion in [7] (p. 51)): consider $\nabla^{1} \in \mathcal{C}_{m}(M)$, with the torsion tensor field of the form $T^{1}=$ $\frac{1}{2} B-\frac{1}{2} B^{\prime}$, for some skew-symmetric tensor field $B \in \mathcal{T}_{2}^{1}(M)$. Let $\nabla$ be a connection on $M$, with torsion $B$. Define $\nabla^{2}:=2 \nabla^{1}-\nabla$. It follows that $T^{2}=-B^{\prime}$. Then, $\nabla^{2}=\nabla^{*}$ and $\nabla^{1}=\frac{1}{2} \nabla+\frac{1}{2} \nabla^{*}$. In conclusion, to any connection $\hat{\nabla} \in \mathcal{C}_{m}(M)$ with the respective special torsion, we can associate an infinite family of dual connections, such that $\hat{\nabla}$ is the mean connection for every element of this family. In particular, this works for the Levi-Civita connection $\nabla^{0}$, because $B^{0}=0$ in this case. (An elementary comparation: in order to identify a closed interval on the real line, we may specify both its ends, or we may specify one of its ends and its middle point.)

The next example shows that we may generalize the previous "arithmetic" mean.

Example 1. Consider an arbitrary couple $\left(\nabla, \nabla^{*}\right)$ of conjugate connections. We define a 1parameter family of connections $\left\{\nabla^{(f)}\right\}_{f \in \mathcal{F}(M)}$, called the $f$-connections, such that $\left(\nabla^{(-f)}, \nabla^{(f)}\right)$ are dually coupled to the metric, where

$$
\nabla^{(f)}:=\frac{1+f}{2} \nabla+\frac{1-f}{2} \nabla^{*}
$$

We get $\nabla^{(f)}=\nabla^{0}+A^{f}$, with

$$
A^{f}:=\frac{1+f}{2} A-\frac{1-f}{2} A^{\prime} \in \mathcal{T}_{2}^{1}(M)
$$

and we obtain a family of deformation algebras $\left(\mathcal{X}(M), A^{f}\right)$.

In particular, we have the $\alpha$-connections $\left\{\nabla^{\alpha}\right\}_{\alpha \in \mathbf{R}}$. These connections generalize the classical ones (see for example $[7,15]$ ), which are symmetric.

In the general case, a short calculation shows that

$$
\left(\nabla_{X}^{(f)} g\right)(Y, Z)=-f g\left(\left(A+A^{\prime}\right)(X, Y), Z\right)
$$

and

$$
2 T^{f}(X, Y)=(1+f)[A(X, Y)-A(Y, X)]-(1-f)\left[A^{\prime}(X, Y)-A^{\prime}(Y, X)\right]
$$

Conversely, start with a connection $\nabla^{1} \in \mathcal{C}(M)$, satisfying (3) and (4) for some $A \in \mathcal{T}_{2}^{1}(M)$. Consider a function $f$ and the connection $\nabla:=\nabla^{0}+A$. Then, there exists a 
unique connection $\nabla^{2}$ such that $\nabla^{*}=\nabla^{2}, \nabla^{(f)}=\nabla^{1}$ and $\left(\nabla, \nabla^{*}\right)$ are conjugate. For $f=0$, we recover the construction in the Remark 2, (v). (An elementary comparation: in order to identify a closed interval on the real line, we may specify both its ends, or we may specify one of its ends and the point which divides the interval in some given "ratio" $f$ ).

Finally, we remark that Formula (3) shows the direct proportionality between the obstruction to the $\nabla^{(f)}$-parallelism of the metric $g$ (on the left side) and the extent to which $\nabla$ differs from $\nabla^{*}$ (i.e., $A+A^{\prime}$ differs from 0 ), weighted through the "conformal factor" $(-f)$.

\section{The Main Geometric Invariants Associated to Dual Connections}

Let $\nabla$ and $\nabla^{*}$ be dual connections on a semi-Riemannian manifold $(M, g)$, with the Levi-Civita connection $\nabla^{0}$. We denote by $T, t, R$, Ric, Far, and $\rho$ the torsion tensor, the "mean torsion" one form, the curvature tensor, the Ricci tensor, the Faraday tensor, and the "pseudo-scalar" curvature of $\nabla$, respectively, defined by

$$
\begin{gathered}
T(X, Y)=\nabla_{X} Y-\nabla_{Y} X-[X, Y], \\
t(X):=\operatorname{trace}(Y \rightarrow T(Y, X)), \\
R(X, Y) Z=\nabla_{X} \nabla_{Y} Z-\nabla_{Y} \nabla_{X} Z-\nabla_{[X, Y]} Z, \\
\operatorname{Ric}(X, Y):=\operatorname{trace}(Z \rightarrow R(Z, X) Y), \\
\operatorname{Far}(X, Y):=\operatorname{trace}(Z \rightarrow R(X, Y) Z)
\end{gathered}
$$

and $\rho=$ traceRic. Similar geometric objects associated to $\nabla^{*}$ and $\nabla^{0}$ will be denoted with an upper $*$ or 0 , respectively. Denote $E_{1}, \ldots, E_{n}$ a local orthonormal basis of vector fields on $(M, g)$.

Remark 3. Using (2), we can determine the previous invariants of $\nabla^{*}$ in terms of $\nabla$ and $g$ :

$$
\begin{array}{r}
g\left(X, T^{*}(Z, Y)\right)=g(X, T(Z, Y))+\left(\nabla_{Z} g\right)(X, Y)-\left(\nabla_{Y} g\right)(X, Z), \\
t^{*}(Y)=t(Y)+\left(\operatorname{div}^{\nabla} g\right)(Y)-\tau(Y),
\end{array}
$$

where we denoted $\tau(Y):=\sum_{i}\left(\nabla_{Y} g\right)\left(E_{i}, E_{i}\right)$,

$$
\begin{array}{r}
g\left(R^{*}(Y, Z) W, X\right)=-g(R(Y, Z) X, W), \\
\operatorname{Ric}^{*}(Z, W)=-\sum_{i} g\left(R\left(E_{i}, Z\right) E_{i}, W\right), \\
\rho^{*}=-\sum_{i, j} g\left(R\left(E_{i}, E_{j}\right) E_{i}, E_{j}\right) .
\end{array}
$$

Remark 4. We denote $A(X, Y):=\nabla_{X} Y-\nabla_{X}^{0} Y$. Then, we can express the invariants of $\nabla$ in terms of $g$ and $A$ :

$$
\begin{gathered}
T(X, Y)=A(X, Y)-A(Y, X) \\
R(X, Y) Z=R^{0}(X, Y) Z+\left(\nabla_{X}^{0} A\right)(Y, Z)-\left(\nabla_{Y}^{0} A\right)(X, Z)+ \\
+A(X, A(Y, Z))-A(Y, A(X, Z)), \\
\operatorname{Ric}(Y, Z)=\operatorname{Ric}^{0}(Y, Z)+\sum_{i}\left[g\left(E_{i},\left(\nabla_{E_{i}}^{0} A\right)(Y, Z)\right)+g\left(E_{i}, A\left(E_{i}, A(Y, Z)\right)\right)-\right. \\
\left.-g\left(E_{i},\left(\nabla_{Y}^{0} A\right)\left(E_{i}, Z\right)\right)-g\left(E_{i}, A\left(Y, A\left(E_{i}, Z\right)\right)\right)\right] .
\end{gathered}
$$

If we denote $A^{t}(X, Y)=A(Y, X)$, then we have the equivalent formula

$$
\operatorname{Ric}(Y, Z)=\operatorname{Ric}^{0}(Y, Z)+\operatorname{div} A(Y, Z)+\operatorname{trace}_{A(Y, Z)}^{t}-\operatorname{trace}\left(\nabla_{Y}^{0} A\right)(Z, \cdot)-\operatorname{trace} A_{Y} \circ A_{Z}^{t} .
$$




$$
\begin{aligned}
\rho=\rho^{0} & +\sum_{i, j}\left[g\left(E_{i},\left(\nabla_{E_{i}}^{0} A\right)\left(E_{j}, E_{j}\right)\right)+g\left(E_{i}, A\left(E_{i}, A\left(E_{j}, E_{j}\right)\right)\right)-\right. \\
& \left.-g\left(E_{i},\left(\nabla_{E_{j}}^{0} A\right)\left(E_{i}, E_{j}\right)\right)-g\left(E_{i}, A\left(E_{j}, A\left(E_{i}, E_{j}\right)\right)\right)\right] .
\end{aligned}
$$

Using coordinates associated to the given orthonormal basis on $M$, one has

$$
R i c_{i k}=R i c_{i k}^{0}+\nabla_{l}^{0} A_{i k}^{l}-\nabla_{i}^{0} A_{l k}^{l}+A_{i k}^{l} A_{m l}^{m}-A_{p k}^{s} A_{i s}^{p} .
$$

The Ricci tensor Ric is symmetric iff

$$
\nabla_{l}^{0} A_{i k}^{l}-\nabla_{i}^{0} A_{l k}^{l}+A_{i k}^{l} A_{m l}^{m}-A_{p k}^{s} A_{i s}^{p}=\nabla_{l}^{0} A_{k i}^{l}-\nabla_{k}^{0} A_{l i}^{l}+A_{k i}^{l} A_{m l}^{m}-A_{p i}^{s} A_{i k}^{p} .
$$

The following result is a simple consequence of the previous formulas and may be considered "folklore".

Proposition 1. Let $\nabla \in \mathcal{C}(M)$. Then, (i) $\nabla \in \mathcal{C}_{c}(M)$ if and only if $T=T^{*}$; (ii) $\nabla \in$ $\mathcal{C}_{c}(M)$ if and only if $\nabla^{*} \in \mathcal{C}_{c}(M)$; (iii) $\nabla \in \mathcal{C}_{s c}(M)$ if and only if $\nabla^{*} \in \mathcal{C}_{s c}(M)$; (iv) if $\bar{\nabla}=\frac{1}{2}\left(\nabla+\nabla^{*}\right)$, then $\bar{\nabla} \in \mathcal{C}_{c}(M) ;(\mathrm{v}) \nabla$ and $\nabla^{*}$ have the same (parameterized) geodesics $\Leftrightarrow A(X, X)+A^{\prime}(X, X)=0, \forall X \in \mathcal{X}(M) \Leftrightarrow$ the tensor field $A+A^{\prime}$ is skew-symmetric. (vi) Let $\nabla \in \mathcal{C}_{s c}(M)$. If $\nabla$ and $\nabla^{*}$ have the same (parameterized) geodesics, then $A=A^{\prime}=0$, i.e., $\nabla=\nabla^{*}=\nabla^{0}$.

Proposition 2. Let $\nabla=\nabla^{0}+A$ and $\nabla^{*}=\nabla^{0}-A^{\prime}$ be dual connections on $(M, g)$. Then, the Bianchi identities for $\nabla$ impose the following conditions uppon the control A:

Bianchi 1

$$
\sum_{X, Y, Z}^{c}\left[\left(\nabla_{Y}^{0} A\right)(X, Y)-\left(\nabla_{X}^{0} A\right)(Z, Y)-\right.
$$

$$
-A(X, A(Z, Y))+A(A(X, Z), Y)-A(A(Y, X), Z)+A(Z, A(Y, X))]=0
$$

Bianchi $2 \sum_{X, Y, Z}^{c}\left[A\left(X, R^{0}(Y, Z) W\right)+\nabla_{X}^{0}\left(\nabla_{Y}^{0} A\right)(Z, W)+A\left(X,\left(\nabla_{Y}^{0} A\right)(Z, W)\right)-\right.$

$$
\begin{gathered}
-\nabla_{X}^{0}\left(\nabla_{Z}^{0} A\right)(Y, W)-A\left(X,\left(\nabla_{Z}^{0} A\right)(Y, W)\right)+\nabla_{X}^{0} A(Y, A(Z, W))+ \\
+A(X, A(Y, A(Z, W)))-\nabla_{X}^{0} A(Z, A(Y, W))-A(X, A(Z, A(Y, W))+ \\
+R^{0}(A(X, Y)-A(Y, X), Z) W+\left(\nabla_{A(X, Y)-A(Y, X)}^{0} A\right)(Z, W)-\left(\nabla_{Z}^{0} A\right)(A(X, Y)- \\
-A(Y, X), W)+A(A(X, Y)-A(Y, X), A(Z, W))- \\
-A(Z, A(A(X, Y)-A(Y, X), W))]=0 .
\end{gathered}
$$

Similar conditions arise for $\nabla^{*}$, replacing $A$ by $-A^{\prime}$.

Remark 5. With the notations in the previous proposition, we deduce a consequence of combining the first Bianchi identity for $\nabla$ and $\nabla^{*}$

$$
\begin{gathered}
\sum_{X, Y, Z}^{c}\left[-g(R(X, Y) W, Z)-g\left(T\left(T^{*}(X, Y), Z\right), W\right)-\right. \\
-\left(\nabla_{T^{*}(X, Y) g}\right)(Z, W)+\left(\nabla_{Z} g\right)\left(T^{*}(X, Y), W\right)- \\
-X\left(g(T(Y, Z), W)-X\left(\left(\nabla_{Y} g\right)(Z, W)\right)+X\left(\left(\nabla_{Z} g\right)(Y, W)\right)+\right. \\
+g\left(T(Y, Z), \nabla_{X} W\right)+\left(\nabla_{Y} g\right)\left(\nabla_{X} W, Z\right)-\left(\nabla_{Z} g\right)\left(\nabla_{X} W, Y\right)+ \\
+g\left(T\left(\nabla_{X}^{*} Y, Z\right), W\right)+\left(\nabla_{\nabla_{X}^{*} Y} g\right)(Z, W)-\left(\nabla_{X} g\right)\left(\nabla_{X}^{*}, Y, W\right)+
\end{gathered}
$$




$$
\left.+g\left(T\left(Y, \nabla_{X}^{*} Z\right), W\right)+\left(\nabla_{Y} g\right)\left(\nabla_{X}^{*} Z, W\right)-\left(\nabla_{\nabla_{X}^{*} Z g}\right)(Y, W)\right]=0 .
$$

The second Bianchi identity leads to a much more complicated relation of compatibility and we omit it.

Theorem 2. Let $\left(\nabla, \nabla^{*}\right)$ be dual connections on the semi-Riemannian manifold $(M, g)$, with $\nabla=$ $\nabla^{0}+A$ and $\nabla^{*}=\nabla^{0}-A^{\prime}$ and let $\gamma=\gamma(t)$ be a geodesic of $g$. Then, the Jacobi fields $J$ along $\gamma$ are solutions of

$$
\begin{aligned}
& \nabla_{\dot{\gamma}} \nabla_{\dot{\gamma}} J+R(J, \dot{\gamma}) \dot{\gamma}-A(T(J, \dot{\gamma}), \dot{\gamma})=\left(\nabla_{J} A\right)(\dot{\gamma}, \dot{\gamma})+A\left(\nabla_{\dot{\gamma}} J, \dot{\gamma}\right)-A(J, A(\dot{\gamma}, \dot{\gamma}))+ \\
+ & A(\dot{\gamma}, A(J, \dot{\gamma}))-A(\dot{\gamma}, A(\dot{\gamma}, J))+\nabla_{\dot{\gamma}} A(\dot{\gamma}, J)-\nabla_{\dot{\gamma}} A(J, \dot{\gamma})+A\left(J, \nabla_{\dot{\gamma}} \dot{\gamma}\right)+A\left(\dot{\gamma}, \nabla_{\dot{\gamma}} J\right) .
\end{aligned}
$$

If, moreover, $\nabla$ is symmetric, then

$$
\nabla_{\dot{\gamma}} \nabla_{\dot{\gamma}} J+R(J, \dot{\gamma}) \dot{\gamma}=\left(\nabla_{J} A\right)(\dot{\gamma}, \dot{\gamma})+2 A\left(\nabla_{\dot{\gamma}} J, \dot{\gamma}\right)-A(J, A(\dot{\gamma}, \dot{\gamma}))+A\left(J, \nabla_{\dot{\gamma}} \dot{\gamma}\right) .
$$

The assertion still holds if we replace $\nabla$ by $\nabla^{*}$ and $A$ by $-A^{\prime}$.

Proof. The Jacobi equation for $J \in \mathcal{X}_{\gamma}$ writes

$$
\nabla_{\dot{\gamma}}^{0} \nabla_{\dot{\gamma}}^{0} J+R^{0}(J, \dot{\gamma}) \dot{\gamma}=0
$$

We use the identity

$$
\begin{gathered}
R^{0}(X, Y) Z=R(X, Y) Z-\left(\nabla_{X} A\right)(Y, Z)+\left(\nabla_{Y} A\right)(X, Z)+ \\
\quad+A(X, A(Y, Z))-A(Y, A(X, Z))-A(T(X, Y), Z) .
\end{gathered}
$$

Replacing $\nabla^{0}$ and $R^{0}$ as functions of $\nabla, R$ and $A$, we get the identity we were looking for.

The previous theorem provides formulas for the transversal control of the geodesics behaviour, expressed in terms of the dual connections instead of the metric. Conversely, we may obtain formulas which express the Jacobi equation along the auto-parallel curves of $\nabla$ or $\nabla^{*}$ (i.e., $\nabla$-"geodesics" or $\nabla^{*}$-"geodesics"), in terms of $g, \nabla^{0}$, and $A$ or $-A^{\prime}$, respectively.

\section{Existence and Characterizations of Statistical Structures}

A triple $(M, g, \nabla)$ is a statistical manifold if $\nabla \in \mathcal{C}_{s c}(M, g)$. In this case, $\left(M, g, \nabla^{*}\right)$ is a statistical manifold too. Alternatively, we denote $\left(M, g, \nabla, \nabla^{*}\right)$ instead of $(M, g, \nabla)$, in order to point out the implicit duality inside.

The centro-affine properties of $\mathcal{C}_{s c}(M)$ w.r.t. $\nabla^{0}$ (together with the metric properties) constitute the geometrical core of the theory of statistical manifolds.

Remark 6. Given the semi-Riemannian manifold $(M, g)$, we point out these five (equivalent) characterizations of a statistical manifold $(M, g, \nabla)$ :

(I)-by relation (1) and $T^{\nabla}=0$.

(II)—through $A \in \mathcal{T}_{2}^{1}(M)$ such that $\nabla=\nabla^{0}+A$, with

$$
g(A(X, Z), Y)=g(A(Y, Z), X), A(X, Y)=A(Y, X) .
$$

(III)—through $T^{\nabla}=0$ and $B \in \mathcal{T}_{3}^{0}(M)$ such that

$$
\left(\nabla_{X} g\right)(Y, Z)=B(X, Y, Z), B(X, Y, Z)=B(Y, X, Z) .
$$

(IV)—through the dual connection $\nabla^{*}$ in (2), such that $T^{\nabla}=T^{\nabla^{*}}=0$.

$(V)$-through the dual connection $\nabla^{*}$ in (2), such that both $A, A^{\prime}$ are symmetric. 
The set of all the invariant statistical structures on a Lie group $G$ will be characterized in Section 6. The Lie algebra $L(G)$ will allow us to "count" easier "how many" statistical structures exist on $G$.

Example 2. (classical statistical manifolds, i.e., for dual connections without torsion) Consider a fixed $n$-dimensional semi-Riemannian manifold $(M, g)$. We have the canonical (and trivial) structure of statistical manifold $\left(M, g, \nabla^{0}\right)$, with $\left(\nabla^{0}\right)^{*}=\nabla^{0}$. The set of all the statistical structures on $(M, g)$ is parameterized by $\mathcal{C}_{s c}(M, g)$. This is a large set (see Section 6$)$ and the (1,2)-type deformation tensor fields measure "how far" a statistical structure is from the canonical one. In what follows, we construct particular new statistical structures on $(M, g)$, in a down-to-up hierarchical way.

(i) Let fix $\xi \in \mathcal{X}(M)$ and denote $\xi^{b}:=g(\xi, \cdot)$ its dual 1-form w.r.t. $g$. Define $A_{\xi}(X, Y)=\xi^{b}(X) Y+\xi^{b}(Y) X+g(X, Y) \xi$ and $\nabla:=\nabla^{0}+A_{\xi}$. Then, $\nabla \in \mathcal{C}_{s c}(M, g)$ and $\nabla^{*}=\nabla^{0}-A_{\xi}$. Thus, on each semi-Riemannian manifold, there always exists an infinite family of (distinct) dual connections, each of them corresponding to a different statistical structure associated to $(M, g)$.

(ii) Suppose $M$ is parallelizable and consider a fixed basis $\left\{E_{1}, \ldots, E_{n}\right\}$ in $\mathcal{X}(M)$. We denote $\stackrel{i}{\nabla}:=\nabla^{0}+A_{E_{i}}$, as defined in (i). We have $n$ "independent" statistical structures $(M, g, \stackrel{i}{\nabla})$ with $i=\overline{1, n}$. Moreover, each affine combination of these connections provides a new statistical structure, a "mean" with specified weights, which may control the global measuring in a specific way (w.r.t. the fixed basis, of course).

(iii) If $M$ is not parallelizable, we may consider (if any) a linearly independent set $\left\{E_{1}, \ldots, E_{k}\right\}$ in $\mathcal{X}(M), k<n$, and make a similar construction as in (ii).

(iv) Let fix $F \in \mathcal{T}_{1}^{1}(M)$ and denote $F^{\prime}$ its adjoint w.r.t. $g$, i.e., $g(F X, Y)=g\left(X, F^{\prime} Y\right)$. Fix $\alpha, \beta \in \mathcal{T}_{1}^{0}(M)$ and denote $\alpha^{\#}, \beta^{\#}$ their dual vector fields w.r.t. $g$, i.e., $g\left(\alpha^{\#}, X\right)=\alpha(X)$ and $g\left(\beta^{\#}, X\right)=\beta(X)$. Fix two symmetric $\omega, \eta \in \mathcal{T}_{2}^{0}(M), \xi \in \mathcal{X}(M)$, define

$$
A(X, Y)=\alpha(X) Y+\alpha(Y) X+\beta(X) F Y+\beta(Y) F X+\omega(X, Y) \xi+\eta(X, Y) F \xi
$$

and $\nabla:=\nabla^{0}+A$. Then $\nabla$ is a symmetric connection and $\nabla^{*}=\nabla^{0}-A^{\prime}$, where

$$
\begin{aligned}
A^{\prime}(X, Y)=\alpha & (X) Y+g(X, Y) \alpha^{\#}+\beta(X) F^{\prime} Y+g(F X, Y) \beta^{\#}+ \\
& +g(\xi, Y) \tilde{\omega}(X)+g\left(\xi, F^{\prime} Y\right) \tilde{\eta}(X)
\end{aligned}
$$

and $\tilde{\omega}, \tilde{\eta} \in \mathcal{T}_{1}^{1}(M)$ are given by $g(\tilde{\omega}(X), Y)=\omega(X, Y)$ and $g(\tilde{\eta}(X), Y)=\eta(X, Y)$.

We have $\nabla \in \mathcal{C}_{S C}(M, g)$ if and only if

$$
\begin{aligned}
& \alpha(Z) Y+\beta(Z) F^{\prime} Y+g(F Z, Y) \beta^{\#}+\xi^{b}(Y) \tilde{\omega}(Z)+\xi^{b}\left(F^{\prime} Y\right) \tilde{\eta}(Z)= \\
& =\alpha(Y) Z+\beta(Y) F^{\prime} Z+g(F Y, Z) \beta^{\#}+\xi^{b}(Z) \tilde{\omega}(Y)+\xi^{b}\left(F^{\prime} Z\right) \tilde{\eta}(Y) .
\end{aligned}
$$

In particular, it follows that

$$
\begin{gathered}
(n-1) \alpha(Y)=2 \beta\left(F^{\prime} Y\right)-\beta(F Y)-\beta(Y) \text { trace } F^{\prime}+ \\
+\xi^{b}(Y) \operatorname{trace} \tilde{\omega}+\xi^{b}(F Y) \operatorname{trace} \tilde{\eta}-\xi^{b}(\tilde{\omega}(Y))-\xi^{b}(F \tilde{\eta}(Y))
\end{gathered}
$$

and

$$
(n-1) \alpha^{\#}=2 F \beta^{\#}-F^{\prime} \beta^{\#}-\operatorname{trace} F^{\prime} \beta^{\#}+\operatorname{trace} \tilde{\omega} \xi+\operatorname{trace} \tilde{\eta} F^{\prime} \xi-\tilde{\omega}^{\prime}(\xi)-\tilde{\eta}^{\prime}\left(F^{\prime} \xi\right) .
$$

Relation (8) may be viewed as an equation in the unknowns $\alpha, \beta, \xi, F, \omega, \eta$, which always has solutions, as $\alpha$ depends explicitly on the variables from the right side.

We distinguish the following special cases: 
(iv) $)_{1}$ Suppose $F=0$. Then,

$$
A(X, Y)=\alpha(X) Y+\alpha(Y) X+\omega(X, Y) \xi
$$

and (8) writes $(n-1) \alpha^{\#}=\operatorname{trace}(\tilde{\omega}) \xi-\tilde{\omega}^{\prime}(\xi)$. In particular, for $\xi=\alpha^{\#}$ and $\omega=g$ we get the examples from the family (iii).

(iv) $)_{2}$ Suppose $F=I d$. Then,

$$
A(X, Y)=(\alpha(X)+\beta(X)) Y+(\alpha(Y)+\beta(Y)) X+(\omega(X, Y)+\eta(X, Y)) \xi
$$

and (8) writes

$$
(n-1) \alpha^{\#}=(1-n) \beta^{\#}+\operatorname{trace}(\tilde{\omega}) \xi-\tilde{\omega}^{\prime}(\xi)+\operatorname{trace}(\tilde{\eta}) \xi-\tilde{\eta}^{\prime}(\xi) .
$$

(iv) $)_{3}$ If $\omega=\eta=0$, then

$$
A(X, Y)=\alpha(X) Y+\alpha(Y) X+\beta(X) F Y+\beta(Y) F X .
$$

We get

$$
(n-1) \alpha^{\#}=2 F \beta^{\#}-F^{\prime} \beta^{\#}-\operatorname{trace}^{\prime} \beta^{\#} .
$$

(iv) $)_{4}$ If $\xi=\alpha^{\#}, \omega=g$, then

$$
A(X, Y)=\alpha(X) Y+\alpha(Y) X+\beta(X) F Y+\beta(Y) F X+g(X, Y) \alpha^{\#}+\eta(X, Y) F \alpha^{\#} .
$$

We get

$$
2 F \beta^{\#}-F^{\prime} \beta^{\#}-\operatorname{trace} F^{\prime} \beta^{\#}+\operatorname{trace} \tilde{\eta} F^{\prime} \alpha^{\#}-\tilde{\eta}^{\prime}\left(F^{\prime} \alpha^{\#}\right)=0 .
$$

(a) If, moreover, $\eta=-g$, then

$$
2 F \beta^{\#}-F^{\prime} \beta^{\#}-\operatorname{trace}^{\prime} \beta^{\#}-(n-1) F^{\prime} \alpha^{\#}=0 .
$$

In particular, if $\alpha=\beta$, then

$$
2 F \alpha^{\#}-\operatorname{traceF}^{\prime} \alpha^{\#}-n F^{\prime} \alpha^{\#}=0 .
$$

(b) If, moreover, $\eta=g$, then

$$
2 F \beta^{\#}-F^{\prime} \beta^{\#}-\operatorname{trace}^{\prime} \beta^{\#}+(n-1) F^{\prime} \alpha^{\#}=0 .
$$

In particular, if $\alpha=\beta$, then

$$
2 F \alpha^{\#}-\operatorname{trace}^{\prime} \alpha^{\#}+(n-2) F^{\prime} \alpha^{\#}=0 .
$$

(iv) $)_{5}$ If $\xi=\alpha^{\#}, \omega=-g$, then

$$
A(X, Y)=\alpha(X) Y+\alpha(Y) X+\beta(X) F Y+\beta(Y) F X-g(X, Y) \alpha^{\#}+\eta(X, Y) F \alpha^{\#} .
$$

We get

$$
2(n-1) \alpha^{\#}=2 F \beta^{\#}-F^{\prime} \beta^{\#}-\operatorname{trace}^{\prime} \beta^{\#}+\operatorname{trace} \tilde{\eta} F^{\prime} \alpha^{\#}-\tilde{\eta}^{\prime}\left(F^{\prime} \alpha^{\#}\right) .
$$

(a) If, moreover, $\eta=g$, then

$$
2(n-1) \alpha^{\#}=2 F \beta^{\#}-F^{\prime} \beta^{\#}-\operatorname{traceF}^{\prime} \beta^{\#}+(n-1) F^{\prime} \alpha^{\#} .
$$

In particular, if $\alpha=\beta$, then

$$
2(n-1) \alpha^{\#}=2 F \alpha^{\#}-\operatorname{traceF}^{\prime} \alpha^{\#}+(n-2) F^{\prime} \alpha^{\#} .
$$


(b) If, moreover $\eta=-g$, then

$$
2(n-1) \alpha^{\#}=2 F \beta^{\#}-F^{\prime} \beta^{\#}-\operatorname{trace}^{\prime} \beta^{\#}-(n-1) F^{\prime} \alpha^{\#} .
$$

In particular, if $\alpha=\beta$, then

$$
2(n-1) \alpha^{\#}=2 F \alpha^{\#}-\operatorname{trace}^{\prime} \alpha^{\#}-n F^{\prime} \alpha^{\#} .
$$

All these examples show that, on every semi-Riemannian manifold, there always exist many families of (distinct) dual connections; the choice of the parameters $\alpha, \beta, F, \omega, \eta$ allows a large flexibility and variability of the possible associated statistical models.

(v) Let $f \in \mathcal{F}(M)$ and $\nabla \in \mathcal{C}_{\mathcal{S}}(M)$ such that $d f\left(R^{\nabla}(X, Y) Z\right)=0$ and $g:=\operatorname{Hess}_{f}^{\nabla}$ is non-degenerated. Then $(M, g, \nabla)$ is a statistical manifold. Here, we used (1) and

$$
\left(\nabla_{X} \operatorname{Hess}_{f}^{\nabla}\right)(Y, Z)-\left(\nabla_{Y} \operatorname{Hess}_{f}^{\nabla}\right)(X, Z)=-R^{\nabla}(X, Y) Z(f) .
$$

(We remark that the hypothesis is much weaker than imposing the curvature flatness of $\nabla$.) The dual connection of $\nabla$ is uniquely determined by

$$
\left(\nabla_{X} d f\right)\left(\nabla_{Z}^{*} Y\right)=Z\left\{\left(\nabla_{X} d f\right) Y\right\}-\left(\nabla_{Y} d f\right)\left(\nabla_{Z} X\right) .
$$

In particular, if $f$ is a divergence function associated to a parameterized family of distributions of probability, then $g$ is the Fisher metric associated to it.

Remark 7. In [7], p. 180, the authors suggest some open problems of interest. The third one is the following:

"Let $(M, g)$ be a Riemannian space. We say that $(M, g)$ may be flattened if there exists a pair of affine connections $\nabla$ and $\nabla^{*}$ such that $\left(M, g, \nabla, \nabla^{*}\right)$ is dually flat. Show whether this is always possible. If not, find the invariant which characterizes those spaces which may be flattened."

From (6) we see that $R^{\nabla}=0$ if and only if $R^{\nabla^{*}}=0$. Suppose that $T^{\nabla}=T^{\nabla^{*}}=0$. The existence of a dually flat structure $\left(M, g, \nabla, \nabla^{*}\right)$ is then equivalent, in this case, with the existence of an affine structure on $M$, which is a longstanding open problem in Differential Geometry. For example, there exist Lie groups which do not admit such a left invariant structure.

If we relax the hypothesis, and accept $\nabla$ and $\nabla^{*}$ have torsion, then we obtain the following characterization of the spaces which may be flattened.

Theorem 3. (i) Let $\left(M, g, \nabla, \nabla^{*}\right)$ be a semi-Riemannian space with a pair of flat dual connections, not necessarily symmetric. Then $M$ is parallelizable.

(ii) Conversely, suppose $(M, g)$ is a parallelizable semi-Riemannian space. Then, there exists a pair of flat dual connections $\left(\nabla, \nabla^{*}\right)$, compatible with $g$.

Proof. (i) Because $\nabla$ is flat, it follows that $M$ is a parallelizable manifold. This is a purely affine differential result, which has nothing to do with the semi-Riemannian structure of the space. Furthermore, it does not involve some properties related to the torsion, for example the eventual symmetry of the connection.

(ii) Consider $E_{1}, \ldots, E_{n}$ a global basis of vector fields on $(M, g)$. Let $\nabla^{-}$and $\nabla^{+}$ be the Cartan-Schouten connections on $M$, defined by $\nabla_{E_{i}}^{-} E_{j}=0$ and $\nabla_{E_{i}}^{+} E_{j}=\left[E_{i}, E_{j}\right]$, respectively, for every indices $i, j=\overline{1, n}$. Then,

$$
\nabla_{X}^{-} Y=X\left(Y^{j}\right) E_{j}, \nabla_{X}^{+} Y=X\left(Y^{j}\right) E_{j}+X^{i} Y^{j}\left[E_{i}, E_{j}\right],
$$


where $X=X^{i} E_{i}, Y=Y^{j} E_{j}$ are arbitrary vector fields on $M$. One knows that $R^{\nabla^{-}}=R^{\nabla^{+}}=0$; in general, both connections have non-null torsion. We define $\left(\nabla^{-}\right)^{*}$ and $\left(\nabla^{+}\right)^{*}$, using (2). In the chosen frame, their coefficients are given, respectively, by

$$
\left(\Gamma^{-}\right)^{*}{ }_{i k}^{s}=E_{i}\left(g_{j k}\right) g^{j s},\left(\Gamma^{+}\right)^{* s}{ }_{i k}=E_{i}\left(g_{j k}\right) g^{j s}-\left[E_{i}, E_{j}\right]^{l} g_{l k} g^{j s} .
$$

Then, $\left(M, g, \nabla^{-},\left(\nabla^{-}\right)^{*}\right)$ and $\left(M, g, \nabla^{+},\left(\nabla^{+}\right)^{*}\right)$ are dually flat. It is possible that, in some cases, these two structures coincide.

Remark 8. The proof of the second part of the previous theorem suggests the following question: on which parallelizable semi-Riemannian manifold does there exist a dually flat structure which, moreover, has both connections with parallel torsion? The Lie groups endowed with left-invariant semi-Riemannian metrics are the first candidates, as then $\nabla^{-}$has $\nabla^{-}$-parallel torsion (see also Section 6).

\section{Beyond the Beaten Path: Exotic Statistical-Like Manifolds}

The classical statistical manifolds $\left(M, g, \nabla, \nabla^{*}\right)$ with symmetric dual connections were generalized to statistical manifolds with torsion, in the works of Kurose and Matsuzoe $[9,16]$ and denoted under the acronym SMAT. They satisfy

$$
\left(\nabla_{Z} g\right)(X, Y)-\left(\nabla_{Y} g\right)(X, Z)=-g(T(Z, Y), X) .
$$

In the following, we define nine new similar families of generalized statistical manifolds (with torsion), denoted $\mathrm{SMAT}_{i}$, for $i=\overline{1,9}$.

Definition 1. Let $(M, g)$ be a semi-Riemannian manifold and $\left(\nabla, \nabla^{*}\right)$ dual connections. Let $\xi$ be an arbitrary fixed vector field and $f_{1}, f_{2}$ fixed functions on $M$. The structure $\left(M, g, \nabla, \nabla^{*}\right)$ is called statistical manifold with torsion of type $i$, for $i=\overline{1,9}$, if

$$
\begin{aligned}
& \left(\nabla_{Z} g\right)(X, Y)-\left(\nabla_{Y} g\right)(X, Z)=g(R(Z, Y) X, \xi), \\
& \left(\nabla_{Z} g\right)(X, Y)-\left(\nabla_{Y} g\right)(X, Z)=\{\operatorname{Ric}(Z, Y)-\operatorname{Ric}(Y, Z)\} \xi^{b}(X) \\
& \left(\nabla_{Z} g\right)(X, Y)-\left(\nabla_{Y} g\right)(X, Z)=g(A(Z, Y)-A(Y, Z), X), \\
& \left(\nabla_{Z} g\right)(X, Y)-\left(\nabla_{Y} g\right)(X, Z)=f_{1} g(T(Z, Y), X)+f_{2} g(R(Z, Y) X, \xi), \\
& \left(\operatorname{div}^{\nabla} g\right)(Y)=\operatorname{trace} \nabla_{Y} g \\
& \left(\operatorname{div} \nabla_{g}\right)(Y)=\operatorname{trace} \nabla_{Y} g-\operatorname{Far}(Y, \xi) \\
& \left(\operatorname{div}^{\nabla} g\right)(Y)=\operatorname{trace}_{Y} \nabla_{Y}-\operatorname{Ric}^{*}(Y, \xi), \\
& \left(\operatorname{div} \nabla_{g}\right)(Y)=\operatorname{trace} \nabla_{Y} g+\operatorname{Ric}(\xi, Y)-\operatorname{Ric}(Y, \xi) \\
& \left(\operatorname{div}^{\nabla} g\right)(Y)=\operatorname{trace}_{Y} g+f_{1} t(Y)-f_{2} \operatorname{Ric}^{*}(Y, \xi)
\end{aligned}
$$

Remark 9. (i) A necessary and sufficient condition for $\left(S M A T_{1}\right)$ is

$$
g\left(X, T^{*}(Z, Y)-T(Z, Y)\right)=-g\left(X, R^{*}(Z, Y) \xi\right)
$$


We rewrite it as

$$
\begin{gathered}
g(A(Z, X), Y)+g(X, A(Z, Y))-g(A(Y, X), Z)- \\
-g(X, A(Y, Z))=-g\left(R^{0}(Z, Y) X, \xi\right)-g\left(\left(\nabla_{Z}^{0} A\right)(Y, X), \xi\right)+ \\
+g\left(\left(\nabla_{Y}^{0} A\right)(Z, X), \xi\right)-g(A(Z, A(Y, X)), \xi)+g(A(Y, A(Z, X)) \xi) .
\end{gathered}
$$

These manifolds generalize the statistical manifolds with symmetric and flat dual connections. A non-trivial example is for flat dual connections with the same non-null torsion tensor field.

(ii) A necessary and sufficient condition for $\left(S M A T_{2}\right)$ is

$$
g\left(X, T^{*}(Z, Y)-T(Z, Y)\right)=g(X, \xi)(\operatorname{Ric}(Z, Y)-\operatorname{Ric}(Y, Z))
$$

We rewrite it as

$$
\begin{gathered}
\quad-g(A(Z, X), Y)-g(X, A(Z, Y))+g(A(Y, X), Z)+g(X, A(Y, Z))= \\
=\sum_{i}\left(g\left(E_{i},\left(\nabla_{E_{i}}^{0} A\right)(Z, Y)-\left(\nabla_{E_{i}}^{0} A\right)(Y, Z)\right)-g\left(E_{i},\left(\nabla_{Z}^{0} A\right)\left(E_{i}, Y\right)-\left(\nabla_{Y}^{0} A\right)\left(E_{i}, Z\right)+\right.\right. \\
\left.+g\left(E_{i}, A\left(E_{i}, A(Z, Y)-A(Y, Z)\right)\right)-g\left(E_{i}, A\left(Z, A\left(E_{i}, Y\right)\right)-A\left(Y, A\left(E_{i}, Z\right)\right)\right)\right) \xi^{b}(X) .
\end{gathered}
$$

(iii) A necessary and sufficient condition for $\left(S M A T_{3}\right)$ is

$$
g\left(X, T^{*}(Z, Y)-T(Z, Y)\right)=g(X, A(Z, Y)-A(Y, Z)) .
$$

We rewrite it as

$$
-g(A(Z, X), Y)+g(A(Y, X), Z)-2 g(X, A(Z, Y))+2 g(X, A(Y, Z))=0
$$

(iv) A necessary and sufficient condition for $\left(S M A T_{4}\right)$ is

$$
g\left(X, T^{*}(Z, Y)-T(Z, Y)\right)=f_{1} g(X, T(Z, Y))+f_{2} g\left(X,-R^{*}(Z, Y) \xi\right)
$$

We rewrite it as

$$
\begin{gathered}
-g(A(Z, X), Y)-g(X, A(Z, Y))+g(A(Y, X), Z)+g(X, A(Y, Z))= \\
=f_{1}[g(A(Z, Y), X)-g(A(Y, Z), X)]+f_{2}\left[g\left(R^{0}(Z, Y) X, \xi\right)+g\left(\left(\nabla_{Z}^{0} A\right)(Y, X), \xi\right)-\right. \\
\left.-g\left(\left(\nabla_{Y}^{0} A\right)(Z, X), \xi\right)+g(A(Z, A(Y, X)), \xi)-g(A(Y, A(Z, X)), \xi)\right] .
\end{gathered}
$$

(v) A necessary and sufficient condition for $\left(S M A T_{5}\right)$ is $t=t^{*}$. We rewrite it as

$$
\sum_{i}\left[g\left(A\left(E_{i}, E_{i}\right), Y\right)+g\left(E_{i}, A\left(E_{i}, Y\right)-2 A\left(Y, E_{i}\right)\right)\right]=0 .
$$

Moreover,

(v) ${ }_{1}$ If $\nabla \in \mathcal{C}_{c}(M, g)$, then $\nabla$ is $\operatorname{SMAT}_{5}$.

(v) ${ }_{2}$ Let $\nabla=\nabla^{0}+A, A(X, Y)=\alpha(X) Y-\alpha(Y) X$, where $\alpha$ is 1 -form. If $\nabla$ is $S M A T_{5}$, then $A=0$.

(vi) A necessary and sufficient condition for $\left(S M A T_{6}\right)$ is

$$
t^{*}(Y)=t(Y)-\sum_{i} g\left(E_{i}, R(Y, \xi) E_{i}\right)
$$

We rewrite

$$
\sum_{i}\left[-g\left(A\left(E_{i}, E_{i}\right), Y\right)-g\left(E_{i}, A\left(E_{i}, Y\right)\right)\right]=\sum_{i}\left[-2 g\left(A\left(Y, E_{i}\right), E_{i}\right)\right)-
$$




$$
\begin{gathered}
-g\left(E_{i}, R^{0}(Y, \xi) E_{i}\right)-g\left(E_{i},\left(\nabla_{Y}^{0} A\right)\left(\xi, E_{i}\right)\right)+g\left(E_{i},\left(\nabla_{\xi}^{0} A\right)\left(Y, E_{i}\right)\right)- \\
\left.-g\left(E_{i}, A\left(Y, A\left(\xi, E_{i}\right)\right)\right)+g\left(E_{i}, A\left(\xi, A\left(Y, E_{i}\right)\right)\right)\right] .
\end{gathered}
$$

(vii) A necessary and sufficient condition for $\left(S M A T_{7}\right)$ is

$$
t^{*}(Y)=t(Y)-\sum_{i} g\left(E_{i}, R^{*}\left(E_{i}, Y\right) \xi\right)
$$

We write it as

$$
\begin{gathered}
\sum_{i}\left[-g\left(A\left(E_{i}, E_{i}\right), Y\right)-g\left(E_{i}, A\left(E_{i}, Y\right)\right)\right]= \\
=\sum_{i}\left[-2 g\left(A\left(Y, E_{i}\right), E_{i}\right)\right)+g\left(R^{0}\left(E_{i}, Y\right) E_{i}+\right. \\
\left.\left.+\left(\nabla_{E_{i}}^{0} A\right)\left(Y, E_{i}\right)-\left(\nabla_{Y}^{0} A\right)\left(E_{i}, E_{i}\right)+A\left(E_{i}, A\left(Y, E_{i}\right)\right)-A\left(Y, A\left(E_{i}, E_{i}\right)\right), \xi\right)\right] .
\end{gathered}
$$

(viii) A necessary and sufficient condition for $\left(S M A T_{8}\right)$ is

$$
t^{*}(Y)=t(Y)+\operatorname{Ric}(\xi, Y)-\operatorname{Ric}(Y, \xi),
$$

i.e.,

$$
t^{*}(Y)=t(Y)+\sum_{i} g\left(E_{i}, R\left(E_{i}, \xi\right) Y-R\left(E_{i}, Y\right) \xi\right) .
$$

We rewrite it as

$$
\begin{gathered}
\sum_{i}\left[-g\left(A\left(E_{i}, E_{i}\right), Y\right)-g\left(E_{i}, A\left(E_{i}, Y\right)\right)\right]=\sum_{i}\left[-2 g\left(A\left(Y, E_{i}\right), E_{i}\right)\right)+ \\
+\operatorname{Ric}^{0}(\xi, Y)+g\left(E_{i},\left(\nabla_{E_{i}}^{0} A\right)(\xi, Y)\right)-g\left(E_{i},\left(\nabla_{\xi}^{0} A\right)\left(E_{i}, Y\right)\right)+ \\
\quad+g\left(A\left(E_{i}, A(\xi, Y)\right), E_{i}\right)-g\left(A\left(\xi, A\left(E_{i}, Y\right)\right), E_{i}\right)- \\
-\operatorname{Ric}^{0}(Y, \xi)-g\left(E_{i},\left(\nabla_{E_{i}}^{0} A\right)(Y, \xi)\right)+g\left(E_{i},\left(\nabla_{Y}^{0} A\right)\left(E_{i}, \xi\right)\right)- \\
\left.-g\left(A\left(E_{i}, A(Y, \xi)\right), E_{i}\right)+g\left(A\left(Y, A\left(E_{i}, \xi\right)\right), E_{i}\right)\right] .
\end{gathered}
$$

(ix) A necessary and sufficient condition for $\left(S M A T_{9}\right)$ is

$$
t^{*}(Y)=\left(1+f_{1}\right) t(Y)-f_{2} \sum_{i} g\left(E_{i}, R^{*}\left(E_{i}, Y\right) \xi\right) .
$$

We rewrite it as

$$
\begin{gathered}
\sum_{i}\left[-g\left(A\left(E_{i}, E_{i}\right), Y\right)-g\left(E_{i}, A\left(E_{i}, Y\right)\right)\right]= \\
=\sum_{i}\left[-2\left(1+f_{1}\right) g\left(A\left(Y, E_{i}\right), E_{i}\right)+g\left(R^{0}\left(E_{i}, Y\right) E_{i}+\right.\right. \\
\left.\left.+\left(\nabla_{E_{i}}^{0} A\right)\left(Y, E_{i}\right)-\left(\nabla_{Y}^{0} A\right)\left(E_{i}, E_{i}\right)+A\left(E_{i}, A\left(Y, E_{i}\right)\right)-A\left(Y, A\left(E_{i}, E_{i}\right)\right), \xi\right)\right] .
\end{gathered}
$$

Remark 10. We have the following inclusions: (i) $\operatorname{SMAT}_{3} \subset \operatorname{SMAT}_{4}\left(f_{1}=1, f_{2}=0\right)$; (ii) $\operatorname{SMAT}_{1} \subset \operatorname{SMAT}_{4}\left(f_{1}=0, f_{2}=1\right)$; (iii) $\operatorname{SMAT}_{7} \subset \operatorname{SMAT}_{9}\left(f_{1}=0, f_{2}=1\right) ;(i v) \mathcal{C}_{c}(M, g) \subset$ $\operatorname{SMAT}_{5} ;$ (v) $\operatorname{SMAT}_{i} \cap \mathcal{C}_{\mathcal{c}}(M, g) \neq \varnothing$.

Examples of some $\mathrm{SMAT}_{i}{ }^{\prime} \mathrm{s}$ will be given in Section 7.

\section{Invariant Statistical Structures on Lie Groups}

Let $G$ be a $n$-dimensional Lie group and $L(G)$ its Lie algebra. A left invariant statistical structure on $G$ is defined by a left invariant semi-Riemannian metric $g$ and a left invariant connection $\nabla$ satisfying (1). A similar definition works for right invariant statistical structures. A statistical structure is called bi-invariant if it is simultaneously left and right 
invariant. Linearity of the tensorial relations allows simpler expressions of the characteristic properties, as acting on invariant vector fields. For example, for a left invariant statistical structure, relation (1) is equivalent to

$$
g\left(\nabla_{Z} X, Y\right)+g\left(X, \nabla_{Z} Y\right)=g\left(\nabla_{Y} X, Z\right)+g\left(X, \nabla_{Y} Z\right)
$$

for all $X, Y, Z \in L(G)$ and (2) is equivalent to

$$
g\left(\nabla_{Z}^{*} X, Y\right)+g\left(X, \nabla_{Z} Y\right)=0
$$

for all $X, Y, Z \in L(G)$.

The simplest (and trivial) example of bi-invariant statistical structure is given by a biinvariant semi-Riemannian metric together with its Levi-Civita connection $\nabla_{X}^{0} Y=\frac{1}{2}[X, Y]$, for all $X, Y \in L(G)$. The real "line" $\left\{\lambda \nabla^{0} \mid \lambda \in \mathbb{R}\right\}$ contains only bi-invariant connections, so the dimension of the space of bi-invariant connections is at least 1 .

On a $n$-dimensional abelian Lie group $G$, any left invariant geometrical object is also bi-invariant. As the set of symmetric left invariant connections may be identified with the set of symmetric type $(1,2)$ tensors on $L(G)$, it follows that, in this case, there exist plenty of bi-invariant statistical structures on $G$, different from the (previous) trivial ones.

The situation changes drastically as soon as we quit the abelian realm.

Proposition 3. Let $g$ be a bi-invariant semi-Riemannian metric on a compact simple Lie group G. Any bi-invariant statistical structure $(G, g, \nabla)$ is trivial, with the exception of $\operatorname{SU}(n)$, for $n \geq 3$, which admits an infinite family corresponding to

$$
\nabla_{X}^{\alpha} Y=\frac{1}{2}[X, Y]+\alpha\left\{X Y+Y X-\frac{2}{n} \operatorname{tr}(X Y) I\right\} i
$$

for any real number $\alpha$. (By $i$ we denote the imaginary constant.)

Proof. The Levi-Civita connection of $g$ is

$$
\nabla_{X}^{0} Y=\frac{1}{2}[X, Y]=\frac{1}{2}(X Y-Y X)
$$

Consider a symmetric bi-invariant connection $\nabla$ on $G$ such that the triple $(G, g, \nabla)$ be a bi-invariant statistical structure. Then, $\nabla_{X} Y=\frac{1}{2}[X, Y]+A(X, Y)$, for all $X, Y \in L(G)$, where $A$ is a symmetric bi-invariant type $(1,2)$ tensor on $L(G)$.

In [17], it was proven that all the bi-invariant connections on $G$ are trivial, except $S U(n)$ (for $n \geq 3$ ), where there exists a family of connections, depending on two real parameters $v$ and $\mu$, of the form

$$
\nabla_{X}^{\mu, v} Y=\mu[X, Y]+v\left\{X Y+Y X-\frac{2}{n} \operatorname{tr}(X Y) I\right\} i
$$

It follows that $\nabla$ must satisfy (11) for any real number $\alpha$.

Corollary 1. Let $g$ be a bi-invariant semi-Riemannian metric on a compact Lie group $G$. Suppose $L(G)=C(G) \oplus L_{1} \oplus \ldots \oplus L_{q}$, where the center $C(G)$ has dimension $p$ and the $L_{i}$ 's are the simple ideals in $L(G)$. Let $r$ be the number of $\mathfrak{s u}(n)$ 's $(n \geq 3)$ in $L(G)$. Then, the dimension of the space of bi-invariant statistical structures $(G, g, \nabla)$ is given by

$$
\frac{1}{2} p^{2}(p+1)+q(p+1)+r .
$$

Proof. The dimension of the space of all the bi-invariant connections on $G$ was determined in [17] to be

$$
p^{3}+3 p q+q+r
$$


For statistical structures one must restrain to symmetric bi-invariant connections only, which leads to the required number.

In particular, when $G$ is simple, we have $p=0, q=r=1$ and the dimension of the space of bi-invariant symmetric connections is 1 (as stated in Proposition 3).

Corollary 2. Let $g$ be a bi-invariant semi-Riemannian metric on $U(n)(n \geq 3)$. Then, any bi-invariant statistical structure $(U(n), g, \nabla)$ is given by

$$
\begin{gathered}
\nabla_{X} Y=\frac{1}{2}[X, Y]+\alpha(X Y+Y X) i+\beta\{\operatorname{tr}(X) Y+\operatorname{tr}(Y) X\} i+ \\
+\gamma \operatorname{tr}(X Y) i I+\epsilon \operatorname{tr}(X) \operatorname{tr}(Y) i I
\end{gathered}
$$

for arbitrary real numbers $\alpha, \beta, \gamma, \epsilon$.

Proof. For $U(n)$, we have $p=1, q=r=1$ and the dimension of the space of bi-invariant symmetric connections is 4 (as follows from Corollary 1 ).

A basis for the bi-invariant connections on $U(n)$ is given [17] by

$$
\begin{gathered}
\eta_{1}(X, Y)=X Y-Y X, \quad \eta_{2}(X, Y)=(X Y+Y X) i \\
\eta_{3}(X, Y)=i \operatorname{tr}(X) Y, \quad \eta_{4}(X, Y)=i \operatorname{tr}(Y) X \\
\eta_{5}(X, Y)=i \operatorname{tr}(X Y) I, \quad \eta_{6}(X, Y)=i \operatorname{tr}(X) \operatorname{tr}(Y) I,
\end{gathered}
$$

where $I$ is the identity $n \times n$ matrix. As statistical structures involve only symmetric connections, we see that from the "affine connections frame" $\left\{\eta_{1} ; \eta_{2}, \eta_{3}+\eta_{4}, \eta_{5}, \eta_{6}\right\}$ we get that $\nabla-\eta_{1}$ may be uniquely expressed as a combination of $\eta_{2}, \eta_{3}+\eta_{4}, \eta_{5}, \eta_{6}$. (Remember that all the geometric objects here act on the Lie algebra.) We found the required general form of a symmetric bi-invariant connection $\nabla$.

Remark 11. (i) The group $U(1)$ is isomorphic with $S^{1}$, so it admits a unique bi-invariant statistical structure (and that is the trivial one).

(ii) On $U(2)$, the space of bi-invariant statistical structures $(U(2), g, \nabla)$ has only three dimensions, as we have [17] the following relation of linear dependence on $L(G L(2, \mathbb{C}))$

$$
\eta_{2}=\eta_{3}+\eta_{4}+\eta_{5}-\eta_{6}
$$

and thus

$$
\nabla_{X} Y=\frac{1}{2}[X, Y]+\beta\{\operatorname{tr}(X) Y+\operatorname{tr}(Y) X\} i+\gamma \operatorname{tr}(X Y) i I+\epsilon \operatorname{tr}(X) \operatorname{tr}(Y) i I
$$

for arbitrary real numbers $\beta, \gamma, \epsilon$.

(iii) All the symmetric bi-invariant connections on non-abelian compact Lie groups are non-flat, due to a result of Milnor [18].

Proposition 4. Let $G$ be a n-dimensional Lie group, $g$ a left invariant semi-Riemannian metric on $G$. Then, the space of left invariant statistical structures $(G, g, \nabla)$ has the dimension $\frac{1}{6} n(n+$ 1) $(n+2)$.

Proof. Any left invariant connection $\nabla$ may be written

$$
\nabla_{X} Y=\frac{1}{2}[X, Y]+A(X, Y)
$$


where $A$ is a left invariant type $(1,2)$ tensor on $L(G)$. As $\nabla$ must be symmetric and subject to $(7)$, it follows that

$$
A(X, Y)=A(Y, X), g(A(X, Y), Z)=g(A(X, Z), Y),
$$

for all $X, Y, Z \in L(G)$. A simple combinatorics counts the number of independent tensors $A$ to be

$$
n+2 C_{n}^{2}+C_{n}^{3}=\frac{1}{6} n(n+1)(n+2),
$$

which finishes the proof.

Remark 12. (i) Let $G$ be a $n$-dimensional Lie group and $m:=\frac{1}{6} n(n+1)(n+2)$. As a consequence of Proposition 4, we deduce that the set of all the (semi-Riemannian!) left invariant statistical structures $(G, g, \nabla)$ can be parameterized by the direct product of $\mathbb{R}^{m}$ with an open subset of $\mathbb{R}^{\frac{1}{2} n(n+1)}$ (corresponding to the symmetric $n \times n$ non-singular matrices).

(ii) The left invariant connection involved in the previously considered left invariant statistical structures is not supposed to be flat. In this context, flatness would be a very strong restriction, which might forbid the existence of such structures. Moreover, up to now, the existence of flat symmetric left invariant connections on Lie groups is an open problem.

\section{Examples}

In this section, we shall use the framework and notations adapted from [7] (Chapters 2 and 3) and [15], where more details may be found.

Consider $n, m$ positive integers and $M$ a connected $m$-dimensional differentiable manifold. The set $\mathbb{R}^{n} \times M$ is a parametric model for the domain of a family of probability distributions $p: \mathbb{R}^{n} \times M \rightarrow \mathbb{R}, p=p(x, \xi), p(x, \xi)>0, \int p(x, \xi) d x=1$. All integrals have the domain $\mathbb{R}^{n}$. For an arbitrary function $f: \mathbb{R}^{n} \times M \rightarrow \mathbb{R}$, we denote

$$
E_{\xi}[f]:=\int f(x, \xi) p(x, \xi) d x,
$$

with $\xi \rightarrow E_{\xi}[f]$ a function from $M$ to $\mathbb{R}$. We write $\xi=\left(\xi^{1}, \ldots \xi^{m}\right)$ when local coordinates on $M$ are involved. In many applications, $f$ depends on $p$ and the values of the operator $E$ measure some kind of entropy, thus the notation.

Let $l=l(x, \xi): \mathbb{R}^{n} \times M \rightarrow \mathbb{R}, l(x, \xi):=\ln p(x, \xi)$ be the log-likehood function, $S=S_{\xi}: M \rightarrow \mathbb{R}$ the Gibbs entropy function, given by $E_{\xi}[-l]$, i.e.,

$$
S_{\xi}:=-\int l(x, \xi) p(x, \xi) d x .
$$

and $G(\xi):=\left(g_{i j}(\xi)\right)_{i, j=1, m}$ the $m \times m$-matrix of the Fischer Riemannian metric, defined by

$$
g_{i j}(\xi):=\int \partial_{i} l(x, \xi) \partial_{j} l(x, \xi) p(x, \xi) d x,
$$

where we denoted $\partial_{i} l:=\frac{\partial l}{\partial_{\xi^{i}}}$. We have [7]

$$
g_{i j}=E\left[\partial_{i} l \partial_{j} l\right]=-E\left[\partial_{i j} l\right]=\int \frac{1}{p(x, \xi)} \partial_{i} p(x, \xi) \partial_{j} p(x, \xi) d x
$$

Let $\alpha$ be a fixed real number. Then, the connection $\nabla^{(\alpha)}$ from Example 1 has the following coefficients, calculated in a point $\xi \in M$ :

$$
\left(\Gamma_{i j, k}^{(\alpha)}\right)_{\xi}=E_{\xi}\left[\left(\partial_{i} \partial_{j} l+\frac{1-\alpha}{2} \partial_{i} l \partial_{j} l\right) \partial_{k} l\right] .
$$


Here, the coefficients of $\nabla^{(\alpha)}$, with three down indices, are defined by

$$
\Gamma_{i j, k}^{(\alpha)}:=g\left(\nabla_{\partial_{i}}^{(\alpha)} \partial_{j}, \partial_{k}\right)
$$

The coefficients of the Levi-Civita connection of the metric $g$ (also known as the Christoffel coefficients of the first kind) are

$$
\left(\Gamma_{i j, k}^{(0)}\right)_{\xi}=E_{\xi}\left[\left(\partial_{i} \partial_{j} l+\frac{1}{2} \partial_{i} l \partial_{j} l\right) \partial_{k} l\right] .
$$

Whenever it is possible, we shall avoid writing the point $\xi$ in formulas. For example,

$$
\Gamma_{i j, k}^{(\alpha)}=\Gamma_{i j, k}^{(0)}-\frac{\alpha}{2} E\left[\partial_{i} l \partial_{j} l \partial_{k} l\right] .
$$

Example 3. Let $\nabla$ be an arbitrary connection on $M$, given by $\nabla=\nabla^{0}+A$, with $A \in \mathcal{T}_{2}^{1}(M)$. Denote $A_{i j, k}:=g\left(A\left(\partial_{i}, \partial_{j}\right), \partial_{k}\right)$ and $\Gamma_{i j, k}:=\Gamma_{i j, k}^{(0)}+A_{i j, k}$ the coefficients (with three down indices) of a connection $\nabla$.

We shall choose $A$ in order to provide examples for $S M A T_{i}$ 's.

(i) If $A_{i j, k}=E\left[-\frac{1}{2} \partial_{i} l \partial_{j} l \partial_{k} l\right]$, then $\nabla \in \mathcal{C}_{s c}(M, g)$.

(ii) Let $f=f(x, \xi): \mathbb{R}^{n} \times M \rightarrow \mathbb{R}$ be another function providing an entropy function $F=F_{\xi}: M \rightarrow \mathbb{R}$, given by $E_{\xi}[f]$, i.e.

$$
F_{\xi}:=\int f(x, \xi) p(x, \xi) d x .
$$

Many such choices are possible, as many entropy functions were suggested in the last decades (the Tsallis entropy, the von Neumann entropy, the Renyi entropy, etc.) and their various generalizations.

We shall combine the partial derivatives of $l$ and $f$ in $E[]$, in order to get more examples. We consider a generic

$$
\begin{aligned}
A_{i j, k}= & E\left[\partial_{i} \partial_{j}\left(a_{1} f+b_{1} l\right) \partial_{i}\left(a_{2} f+b_{2} l\right)+\partial_{i} \partial_{k}\left(a_{3} f+b_{3} l\right) \partial_{j}\left(a_{4} f+b_{4} l\right)+\right. \\
+ & \partial_{j} \partial_{k}\left(a_{5} f+b_{5} l\right) \partial_{i}\left(a_{6} f+b_{6} l\right)+c_{1} \partial_{i} f \partial_{j} f \partial_{k} f+c_{2} \partial_{i} l \partial_{j} l \partial_{k} l+ \\
& \left.+c_{3} \partial_{i} f \partial_{j} f \partial_{k} l+c_{4} \partial_{i} f \partial_{j} l \partial_{k} l+d_{1} \partial_{i} \partial_{j} \partial_{k} f+d_{2} \partial_{i} \partial_{j} \partial_{k} l\right],
\end{aligned}
$$

where $a_{1}, \ldots, a_{6}, b_{1}, \ldots, b_{6}, c_{1}, \ldots, c_{4}, d_{1}, d_{2}$ are constants to be determined.

(1) If

$$
\begin{array}{ll}
a_{1} a_{2}+2 a_{3} a_{4}-3 a_{5} a_{6}=0, & b_{1} b_{2}+2 b_{3} b_{4}-3 b_{5} b_{6}=0 ， \\
a_{1} b_{2}+2 a_{3} b_{4}-3 a_{5} b_{6}=0 ， & b_{1} a_{2}+2 b_{3} a_{4}-3 b_{5} a_{6}=0 ，
\end{array}
$$

where $c_{i}=0, i=\overline{1,4}, d_{j}=0, j=\overline{1,2}$, then $(M, g, \nabla)$ is $S_{M A T}$.

(2) If

$$
\begin{aligned}
& a_{1} a_{2}-a_{3} a_{4}+2 a_{5} a_{6}=0,3 a_{3} a_{4}-a_{5} a_{6}=0, a_{1} a_{2}+a_{5} a_{6}=0, \\
& b_{1} b_{2}-b_{3} b_{4}+2 b_{5} b_{6}=0,3 b_{3} b_{4}-b_{5} b_{6}=0, b_{1} b_{2}+b_{5} b_{6}=0, \\
& a_{1} b_{2}-a_{3} b_{4}+2 a_{5} b_{6}=0,3 a_{3} b_{4}-a_{5} a_{6}=0, a_{1} b_{2}+a_{5} b_{6}=0, d_{i}=0,1=\overline{1,2}, \\
& b_{1} a_{2}-b_{3} a_{4}+2 b_{5} a_{6}=0,3 b_{3} a_{4}-b_{5} a_{6}=0, b_{1} a_{2}+b_{5} a_{6}=0, c_{i}=0, i=\overline{1,4} .
\end{aligned}
$$

then $(M, g, \nabla)$ is $S_{M A T}$.

For other families of $\operatorname{SMAT}_{i}{ }^{\prime} \mathrm{s}$, the calculations are similar but more tedious. We shall follow now another path, under some more restrictive assumptions. 
Example 4. With the previous notations, let $(M, g)$ have $g_{i j}=\delta_{i j}$, so $\left(\Gamma^{0}\right)_{i j}^{k}=0, \Gamma_{i j}^{k}=\Gamma_{i j, k}=$ $A_{i j, k}$. This may occur, for example, for exponential families of distributions probabilities, i.e., [5,7]

$$
p(x, \xi)=\exp \left\{C(x)+\xi^{i} F_{i}(x)-\psi(x)\right\},
$$

with $\psi(x)=\left(x^{1}\right)^{2}+\ldots+\left(x^{m}\right)^{2}$. Then, we have the following characterizations:

$\operatorname{SMAT}_{1}$

$$
-\Gamma_{k i}^{j}-\Gamma_{k j}^{i}+\Gamma_{j i}^{k}+\Gamma_{j k}^{i}=\left(\Gamma_{j i}^{r} \Gamma_{k r}^{s}-\Gamma_{k i}^{r} \Gamma_{j r}^{s}\right) \xi_{s} .
$$

Let us take $n=2$ and $\xi=\partial_{1}$. One has

$$
\begin{gathered}
-2 \Gamma_{21}^{1}+\Gamma_{11}^{2}+\Gamma_{12}^{1}=\Gamma_{11}^{r} \Gamma_{2 r}^{1}-\Gamma_{21}^{r} \Gamma_{1 r}^{1},-\Gamma_{11}^{2}+\Gamma_{21}^{1}=-\Gamma_{11}^{r} \Gamma_{2 r}^{1}+\Gamma_{21}^{r} \Gamma_{1 r}^{1}, \\
-\Gamma_{12}^{2}+\Gamma_{22}^{1}=\Gamma_{22}^{r} \Gamma_{1 r}^{1}-\Gamma_{12}^{r} \Gamma_{2 r}^{1},-\Gamma_{22}^{1}-\Gamma_{21}^{2}+\Gamma_{12}^{2}+\Gamma_{12}^{1}=\Gamma_{12}^{r} \Gamma_{2 r}^{1}-\Gamma_{22}^{r} \Gamma_{1 r}^{1} .
\end{gathered}
$$

We obtain $\Gamma_{12}^{1}=\Gamma_{21}^{1}=\Gamma_{21}^{2}=a$. Then $T_{12}^{1}=0$. Let us take $T_{12}^{2}=1$. Therefore, $\Gamma_{12}^{2}=1+a$.

If one considers $\Gamma_{11}^{1}=0, \Gamma_{11}^{2}=a-a^{2}, \Gamma_{22}^{2}=\frac{a^{2}-a-1}{a}, a \neq 0, \Gamma_{22}^{1}=0$, then we have one example of connection with torsion for $S M A T_{1}$ and $S M A T_{4}$.

$\operatorname{SMAT}_{2}$

$$
-\Gamma_{k i}^{j}-\Gamma_{k j}^{i}+\Gamma_{j i}^{k}+\Gamma_{j k}^{i}=\sum_{l} E_{l}^{r} E_{l}^{s} \xi_{i}\left[\left(\Gamma_{k j}^{p}-\Gamma_{j k}^{p}\right) \Gamma_{s p}^{r}-\Gamma_{s j}^{p} \Gamma_{k p}^{r}+\Gamma_{s k}^{p} \Gamma_{j p}^{r}\right]
$$

Let $n=2$ and $\xi=\partial_{1}$. If $-\Gamma_{k i}^{j}-\Gamma_{k j}^{i}+\Gamma_{j i}^{k}+\Gamma_{j k}^{i}=0$, we may consider

$$
\left(\Gamma_{12}^{p}-\Gamma_{21}^{p}\right) \Gamma_{s p}^{2}-\Gamma_{s 1}^{p}-\Gamma_{2 p}^{r}+\Gamma_{s 2}^{p} \Gamma_{1 p}^{r}=0,-\Gamma_{s 2}^{p} \Gamma_{2 p}^{r}+\Gamma_{s 2}^{p} \Gamma_{2 p}^{r}=0 .
$$

We get

$$
\begin{gathered}
-2 \Gamma_{21}^{1}+\Gamma_{11}^{2}-\Gamma_{12}^{1}=0,-\Gamma_{11}^{2}+\Gamma_{21}^{1}=0, \\
2 \Gamma_{21}^{2}-\Gamma_{12}^{2}+\Gamma_{22}^{1}=0,-\Gamma_{22}^{1}-\Gamma_{21}^{2}+\Gamma_{12}^{2}+\Gamma_{12}^{1}=0, \\
\Gamma_{22}^{1}-\Gamma_{11}^{1} \Gamma_{21}^{2}-\Gamma_{11}^{2} \Gamma_{22}^{2}+\Gamma_{12}^{1} \Gamma_{11}^{2}+\Gamma_{12}^{2} \Gamma_{21}^{2}=0, \\
\Gamma_{22}^{2}-\Gamma_{21}^{1} \Gamma_{21}^{1}-\Gamma_{21}^{2} \Gamma_{22}^{2}+\Gamma_{22}^{1} \Gamma_{11}^{2}+\Gamma_{22}^{2} \Gamma_{12}^{2}=0 .
\end{gathered}
$$

$\operatorname{SMAT}_{3}$

$$
-\Gamma_{k i}^{j}-2 \Gamma_{k j}^{i}+\Gamma_{j i}^{k}+2 \Gamma_{j k}^{i}=0
$$

In dimension 2 we obtain

$$
\begin{gathered}
-3 \Gamma_{21}^{1}+\Gamma_{11}^{2}+\Gamma_{12}^{1}=0,-\Gamma_{11}^{2}+\Gamma_{21}^{1}=0, \\
3 \Gamma_{21}^{2}-2 \Gamma_{12}^{2}+\Gamma_{22}^{1}=0,-\Gamma_{22}^{1}-2 \Gamma_{21}^{2}+2 \Gamma_{12}^{1}=0 .
\end{gathered}
$$

Let us take the torsion $T_{12}^{1}=1, T_{12}^{2}=0$. We can consider $\Gamma_{21}^{2}=\Gamma_{12}^{2}=a, \Gamma_{12}^{1}=1+\Gamma_{21}^{1}=$ $1+b, \Gamma_{11}^{2}=b, \Gamma_{21}^{1}=\frac{2 b+1}{3}, \Gamma_{22}^{1}=-a$. If $a=2 b+2$ we have one example of affine connection with torsion for $S M A T_{3}$ and $S M A T_{4}$.

$\mathrm{SMAT}_{4}$

$$
-\Gamma_{k i}^{j}-\Gamma_{k j}^{i}+\Gamma_{j i}^{k}+\Gamma_{j k}^{i}=f_{1}\left(\Gamma_{k j}^{i}-\Gamma_{j k}^{i}\right)+f_{2} \xi_{p}\left(\Gamma_{j i}^{r} \Gamma_{k r}^{p}-\Gamma_{k i}^{r} \Gamma_{j r}^{p}\right)
$$

SMAT $_{5}$

$$
\sum_{l} E_{l}^{r} E_{l}^{s}\left(\Gamma_{r s}^{j}+\Gamma_{s j}^{r}-2 \Gamma_{j s}^{r}\right)=0
$$


where $E_{i}=E_{i}^{j} \partial_{j}$. In dimension 2 one can consider $\Gamma_{11}^{2}=\Gamma_{12}^{1}=\Gamma_{21}^{1}=b, \Gamma_{21}^{2}=\Gamma_{12}^{2}=\Gamma_{22}^{1}=$ $a, \Gamma_{11}^{1}=0, \Gamma_{22}^{2}=0$ a solution for $S M A T_{5}$.

$\operatorname{SMAT}_{6}$

$$
\sum_{l} E_{l}^{r} E_{l}^{s}\left[\Gamma_{r s}^{j}+\Gamma_{s j}^{r}-2 \Gamma_{j s}^{r}+\xi^{p}\left(-\Gamma_{p s}^{q} \Gamma_{j q}^{r}+\Gamma_{j s}^{q} \Gamma_{p q}^{r}\right)\right]=0
$$

In dimension 2 , if $\xi=\partial_{1}$, we may consider

$$
\begin{gathered}
\Gamma_{11}^{2}+\Gamma_{12}^{1}-2 \Gamma_{21}^{1}=0, \Gamma_{22}^{1}+\Gamma_{21}^{2}-2 \Gamma_{12}^{2}=0, \Gamma_{12}^{1} \Gamma_{21}^{2}+\Gamma_{22}^{1} \Gamma_{11}^{1}=0, \\
T_{12}^{1}=0, \Gamma_{12}^{2}-\Gamma_{22}^{1}-\Gamma_{12}^{1} \Gamma_{21}^{1}+\Gamma_{22}^{1} \Gamma_{11}^{1}-\Gamma_{12}^{2} \Gamma_{22}^{1}+\Gamma_{22}^{2} \Gamma_{12}^{1}=0, \\
\Gamma_{21}^{1}=\Gamma_{11}^{2},-\Gamma_{12}^{2}+\Gamma_{21}^{2}-\Gamma_{11}^{1} \Gamma_{21}^{2}+\Gamma_{21}^{1} \Gamma_{11}^{2}-\Gamma_{11}^{2} \Gamma_{22}^{2}+\Gamma_{21}^{2} \Gamma_{12}^{2}=0 .
\end{gathered}
$$

$\operatorname{SMAT}_{7}$

$$
\sum_{l} E_{l}^{r} E_{l}^{s}\left[\Gamma_{r s}^{j}+\Gamma_{s j}^{r}-2 \Gamma_{j s}^{r}+\xi_{p}\left(\Gamma_{j s}^{q} \Gamma_{r q}^{p}-\Gamma_{r s}^{q} \Gamma_{j q}^{p}\right)\right]=0
$$

In dimension 2 , if $\xi=\partial_{1}$, one can consider

$$
\begin{gathered}
\Gamma_{11}^{2}+\Gamma_{12}^{1}-2 \Gamma_{21}^{1}+\Gamma_{21}^{2} \Gamma_{12}^{1}-\Gamma_{11}^{2} \Gamma_{22}^{1}=0, \Gamma_{21}^{1}-\Gamma_{11}^{2}+\Gamma_{11}^{2} \Gamma_{22}^{1}-\Gamma_{21}^{2} \Gamma_{12}^{1}=0, \\
\Gamma_{21}^{1}+\Gamma_{21}^{2}-2 \Gamma_{12}^{2}+\Gamma_{12}^{1} \Gamma_{21}^{1}-\Gamma_{22}^{1} \Gamma_{11}^{1}+\Gamma_{12}^{2} \Gamma_{22}^{1}-\Gamma_{22}^{2} \Gamma_{12}^{1}=0, \\
\Gamma_{12}^{1}-\Gamma_{22}^{1}-\Gamma_{12}^{1} \Gamma_{21}^{1}+\Gamma_{22}^{1} \Gamma_{11}^{1}-\Gamma_{12}^{2} \Gamma_{22}^{1}+\Gamma_{22}^{2} \Gamma_{12}^{1}=0, T_{12}^{1}=T_{12}^{2}=0 .
\end{gathered}
$$

$\operatorname{SMAT}_{8}$

$$
\sum_{l} E_{l}^{r} E_{l}^{s}\left[\Gamma_{r s}^{j}+\Gamma_{s j}^{r}-2 \Gamma_{j s}^{r}+\xi^{p}\left(\Gamma_{p j}^{q} \Gamma_{r q}^{s}-\Gamma_{r j}^{q} \Gamma_{p q}^{s}-\Gamma_{j p}^{q} \Gamma_{r q}^{s}+\Gamma_{r p}^{q} \Gamma_{j q}^{s}\right)\right]=0
$$

SMAT $_{9}$

$$
\sum_{l} E_{l}^{r} E_{l}^{s}\left[\Gamma_{r s}^{j}+\Gamma_{s j}^{r}-2 \Gamma_{j s}^{r}-2 f_{1} \Gamma_{j s}^{r}+f_{2} \xi_{p}\left(\Gamma_{j s}^{q} \Gamma_{r q}^{p}-\Gamma_{r s}^{q} \Gamma_{j q}^{p}\right)=0\right.
$$

\section{Discussions}

The paper tries to clarify some notions and results from Differential Geometry, which are motivated by models arising from Statistics, related to statistical manifolds and to dual connections. The main idea is to distinguish, at each level of understanding, which are the appropriate algebraic and/or geometric "controls" for the variability of the models. Thus, we pointed out the deformation algebras $(\mathcal{X}(M), A)$ and the Riemannian Rinehart bi-algebras $(\mathcal{F}(M), \mathcal{X}(M),<,>, \cdot)$, as algebraic invariants underlying behind the dual connections and statistical manifolds.

Second, we characterized the differentiable manifolds admitting dually flat statistical structures with torsion (Theorem 4.4) and proved several results which count the number of statistical manifold structures on compact Lie groups (Section 6).

Third, we define new families of dual connections and of statistical manifolds with and without torsion (including the families $\mathrm{SMAT}_{i}, 1=\overline{1,9}$ ), which impose new assumptions on the curvature and torsion tensor fields. In Section 7 we exemplify them, on particular manifolds of probability distributions.

Several research directions open: (i) the purely algebraic study of the Riemannian Rinehart bi-algebras and of the deformations algebras, associated to specific control tensor fields on statistical manifolds; (ii) the relevance of the $\nabla^{(f)}$-connections for statistics, with arbitrary (or specific) functions $f$, extending the studies when the function is constant; (iii) specific statistical applications for the $\mathrm{SMAT}_{i}$ 's structures; and (iv) optimization results on the space of the control tensors $A$. 
Author Contributions: The authors contributed equally to the writing. All authors have read and agreed to the published version of the manuscript.

Funding: This research received no external funding.

Institutional Review Board Statement: Not applicable.

Informed Consent Statement: Not applicable.

Data Availability Statement: Not applicable.

Acknowledgments: We are grateful to the reviewers for their valuable remarks, which helped us in improving the clarity of the paper.

Conflicts of Interest: The authors declare no conflicts of interest.

\section{References}

1. Amari, S. Differential-Geometrical Methods in Statistics; Lecture Notes in Statistics; Springer: Berlin/Heidelberg, Germany; New York, NY, USA, 1985.

2. Belarbi, O.A.; Meziane, A. Overview and perspectives on metric-affine gravity. J. Phys. Conf. Ser. 2021, 1766, 012007 [CrossRef]

3. Amari, S. Information Geometry and Its Applications; Springer: Tokyo, Japan, 2016; Volume 194.

4. Ay, N.; Jurgen, J.; Le, H.V.; Schwachhöfer, L. Information Geometry; Ergebnisse der Mathematik und ihrer Grenzgebiete, 64; Springer: Heidelberg, Germany; New York, NY, USA, 2017.

5. Calin, O.; Udriste, C. Geometric Modeling in Probability and Statistics; Springer: Heidelberg, Germany; New York, NY, USA, 2014.

6. Nielsen, F. An Elementary Introduction to Information Geometry. Entropy 2020, 22, 1100. [CrossRef] [PubMed]

7. Amari, S.; Nagaoka, H. Methods of Information Geometry; Transl. of Math. Monographs, Amer. Math. Society: Providence, RI, USA, 2000; Volume 191.

8. Kurose, T. Dual connections and affine geometry. Math. Z. 1990, 203, 115-121. [CrossRef]

9. Kurose, T. Statistical Manifolds Admitting Torsion. Geometry and Something; Fukuoka Univ.: Fukuoka-shi, Japan, 2007. (In Japanese)

10. Matsuzoe, H. Statistical manifolds and affine differential geometry. In Probabilistic Approach to Geometry; Advanced Studies in Pure Mathematics; Math. Soc. Japan: Tokyo, Japan, 2010; Volume 57, pp. 303-321.

11. Hirică, I.E.; Nicolescu, L. Associative deformation algebras on Weyl manifolds. Diff. Geom.-Dyn. Syst. 2006, 8, 112-119.

12. Nicolescu, L. Champs des vecteurs remarcables dans l'algebre de deformation; retrospective et perspective. Balk. J. Geom. Appl. 1996, 1, 47-60.

13. Nicolescu, L.; Pripoae, G.T. Structures algebriques engendres par certaines proprietes geometriques. An. Univ. Buc. 1986, 35, 58-64.

14. Pessers, V.; Van der Veken, J. Riemannian manifolds as Lie-Rinehart algebras. Int. J. Geom. Meth. Mod. Phys. 2016, 13, 1641003. [CrossRef]

15. Matsuzoe, H.; Takeuchi, J.; Amari, S. Equiaffine structures on statistical manifolds and Bayesian statistics. Diff. Geom. Appl. 2006, 24, 567-578. [CrossRef]

16. Matsuzoe, H. Information geometry of Bayesian statistics. AIP Conf. Proc. 2015, 279, 1641.

17. Laquer, H.T. Invariant affine connections on Lie groups. Trans. Am. Math. Soc. 1992, 331, 541-551. [CrossRef]

18. Milnor, J. Curvatures of left invariant metrics on Lie groups. Adv. Math. 1976, 21, 293-329. [CrossRef] 